\title{
HIC1 and miR-23 27 24 clusters form a double-negative feedback loop in breast cancer
}

\author{
Yanbo Wang ${ }^{1,6}$, Hongwei Liang ${ }^{1,6}$, Geyu Zhou ${ }^{1,6}$, Xiuting Hu ${ }^{1,6}$, Zhengya Liu ${ }^{1}$, Fangfang Jin ${ }^{1}$, Mengchao Yu ${ }^{1}$, Jianfeng Sang ${ }^{2}$, \\ Yong Zhou ${ }^{3}$, Zheng Fu' ${ }^{1}$, Chen-Yu Zhang ${ }^{1}$, Weijie Zhang ${ }^{\star 4,5}$, Ke Zen ${ }^{\star, 1}$ and Xi Chen ${ }^{\star, 1}$
}

MicroRNAs (miRNAs) have emerged as a major regulator of the initiation and progression of human cancers, including breast cancer. However, the cooperative effects and transcriptional regulation of multiple miRNAs, especially miRNAs that are present in clusters, remain largely undiscovered. Here we showed that all members of the miR-23 $27 \sim 24$ clusters are upregulated and function as oncogenes in breast cancer and simultaneously target HIC1. Furthermore, we found that HIC1 functions as a transcriptional repressor to negatively control the expression of miR-23 $27 \sim 24$ clusters and forms a double-negative (overall positive) feedback loop. This feedback regulatory pathway is important because overexpression of miR-23 27 24 clusters can remarkably accelerate tumor growth, whereas restoration of $\mathrm{HIC} 1$ significantly blocks tumor growth in vivo. A mathematical model was created to quantitatively illustrate the regulatory circuit. Our finding highlights the cooperative effects of miRNAs in a cluster and adds another layer of complexity to the miRNA regulatory network. This study may also provide insight into the molecular mechanisms of breast cancer progression.

Cell Death and Differentiation (2017) 24, 421-432; doi:10.1038/cdd.2016.136; published online 23 December 2016

Breast cancer is the most frequently diagnosed cancer and the leading cause of cancer death in women. ${ }^{1}$ The origin and development of breast cancer remain complex and obscure. ${ }^{2}$ In the past decade, a class of small noncoding RNAs known as microRNAs (miRNAs) has emerged as a major regulator of the initiation and progression of human cancers, including breast cancer. ${ }^{3,4}$ MiRNAs bind target mRNAs at complementary sites in their $3^{\prime}$-untranslated regions (3'-UTRs), thereby suppressing the expression of the target gene at the posttranscriptional level. Dysregulated and dysfunctional miRNAs have a causal role in cancer etiology because miRNAs can regulate targeted oncogenes and tumor suppressors. ${ }^{5-7}$ Further understanding of the molecular functions of miRNAs in breast cancer is urgently needed.

One remarkable aspect of miRNAs is that they are often organized in clusters (within $3 \mathrm{~kb}$ ) in the genome. ${ }^{8}$ Generally, miRNA clusters are transcribed coordinately as polycistronic units that are processed to produce individual miRNAs, resulting in co-expression of the miRNAs. ${ }^{9}$ For example, miR-23 27 24 clusters occupy two genomic loci in humans: the $\mathrm{miR}-23 \mathrm{a} \sim 27 \mathrm{a} \sim 24-2$ cluster is intergenic and produces mature miR-23a, miR-27a and miR-24, whereas the miR$-23 b \sim 27 b \sim 24-1$ cluster is intronic and produces mature miR-23b, miR-27b and miR-24. ${ }^{10}$ However, the reason for this clustering of miRNAs is not fully understood. One intriguing possibility is that the consistent expression of clustered
miRNAs is related to their cooperative effects in regulating specific cellular pathways or deciding the fate of a cell. Unfortunately, most research on miRNAs to date has focused on the roles of individual miRNA in the regulation of specific genes. The overall cellular functions and pathways affected by co-expressed clusters of miRNAs remain largely undiscovered.

Another factor to consider is that miRNAs do not work in isolation but rather act in concert with other classes of regulatory proteins. Transcription factors regulate the transcription of miRNA genes in a manner similar to the regulation of protein-coding genes; that is, by binding to conventional transcription factor binding site sequences located in or near promoter regions upstream of the miRNA genes. ${ }^{11}$ Thus, transcription factors, miRNAs and their respective targets form interconnected feedback and feedforward circuits. ${ }^{12}$ However, only a handful of miRNA transcription factors have been characterized. As a result, it is difficult to decipher the contributions of miRNAs to phenotypic variations and diseases. Thus, the detailed mechanism for transcriptional regulation of miRNAs remains to be elucidated.

In this study, we found that miR-23a $\sim 27 a \sim 24-2$ and miR-23b 27b 24-1 clusters simultaneously target the tumor-suppressor gene hypermethylated in cancer 1 (HIC1). Because HIC1 is a transcriptional repressor, we investigated

\footnotetext{
${ }^{1}$ State Key Laboratory of Pharmaceutical Biotechnology, Jiangsu Engineering Research Center for MicroRNA Biology and Biotechnology, NJU Advanced Institute for Life Sciences (NAILS), School of Life Sciences, Nanjing University, 163 Xianlin Avenue, Nanjing 210046, Jiangsu, China; ${ }^{2}$ Department of Thyroid and Breast Surgery, Affiliated Drum Tower Hospital of Nanjing University Medical School, 321 Zhongshan Road, Nanjing 210008, Jiangsu, China; ${ }^{3}$ Department of Thoracic and Cardiovascular Surgery, Affiliated Drum Tower Hospital of Nanjing University Medical School, 321 Zhongshan Road, Nanjing 210008, Jiangsu, China; ${ }^{4}$ Department of General Surgery, Affiliated Drum Tower Hospital of Nanjing University Medical School, 321 Zhongshan Road, Nanjing 210008, Jiangsu, China and ${ }^{5}$ Department of General Surgery, Drum Tower Clinical College of Nanjing Medical University, 321 Zhongshan Road, Nanjing 210008, Jiangsu, China

*Corresponding author: W Zhang or K Zen or X Chen, School of Life Sciences, Nanjing University, NJU Advanced Institute for Life Sciences (NAILS), 163 Xianlin Avenue, Nanjing, Jiangsu 210046, China. Tel: 86-25-89681323; Fax: 86-25-89681275; E-mail: zhangweijie1616@163.com or kzen@ nju.edu.cn or xichen@nju.edu.cn

${ }^{6}$ These authors contributed equally to this work.

Received 14.6.16; revised 03.10.16; accepted 21.10.16; Edited by A Villunger; published online 23.12.2016
} 

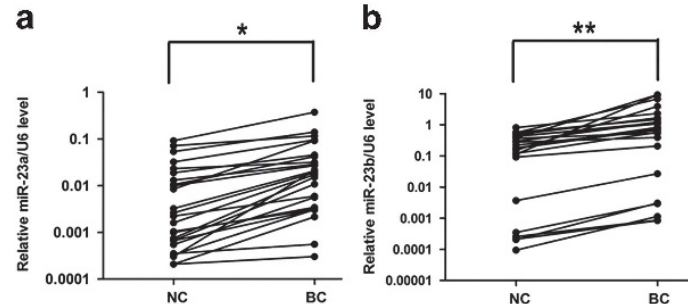

f
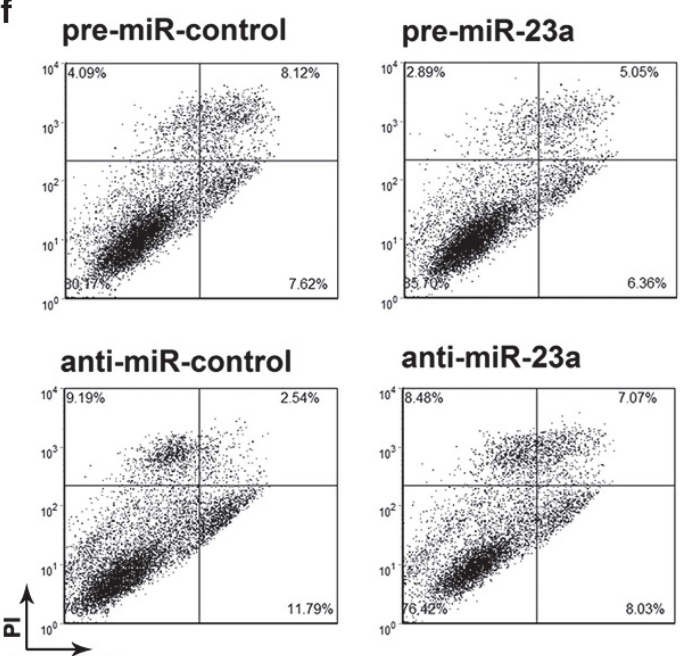

Annexin V

\section{g}
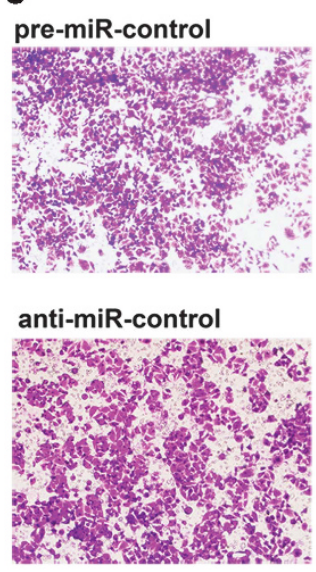
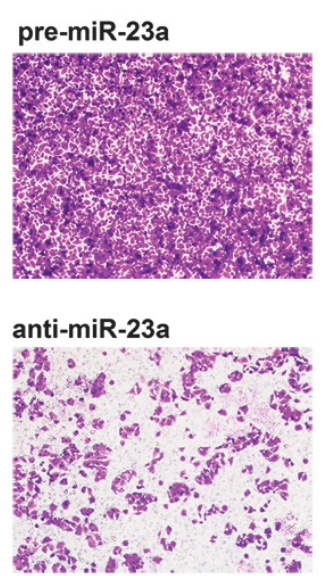
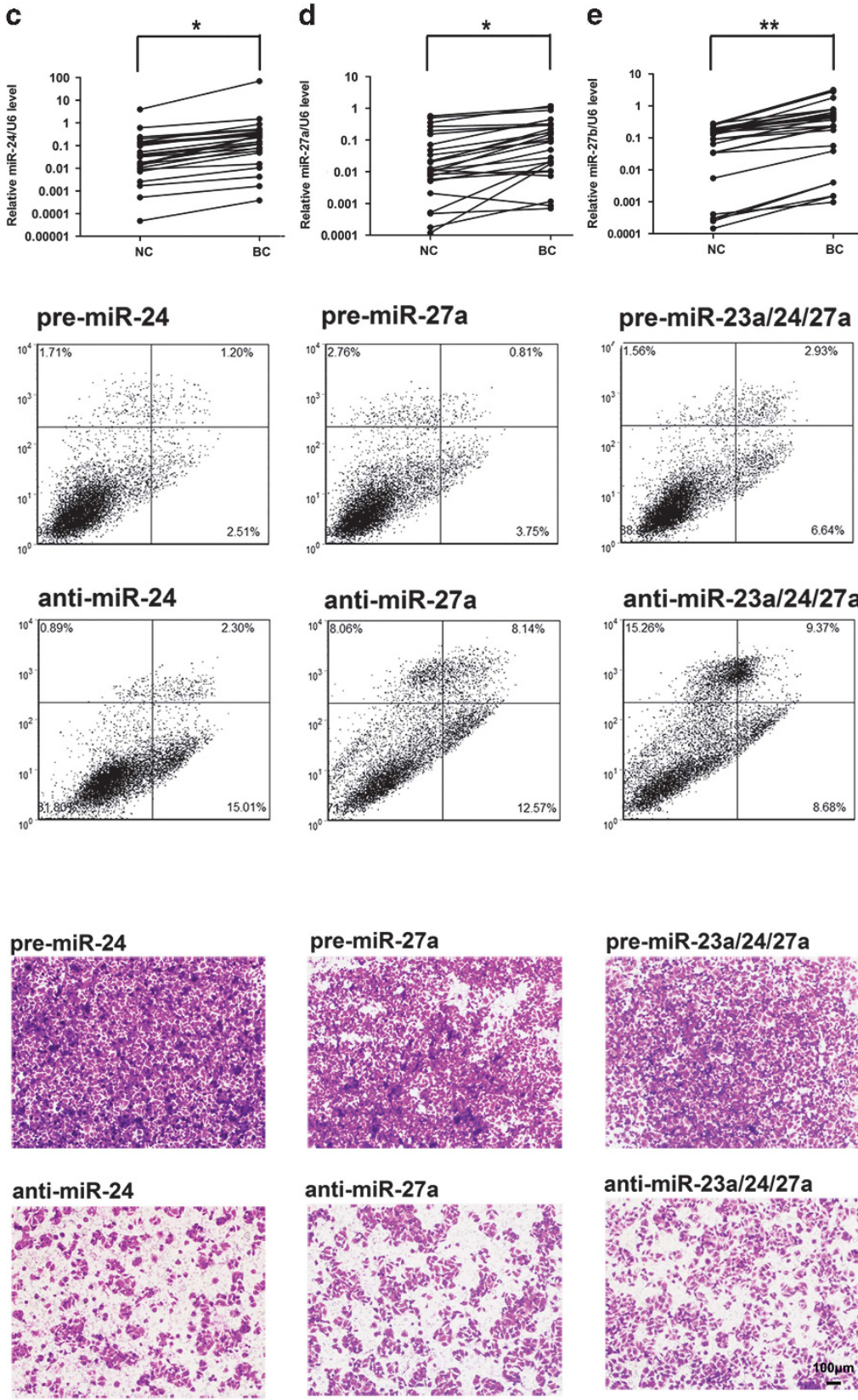

pre-miR-23a/24/27a

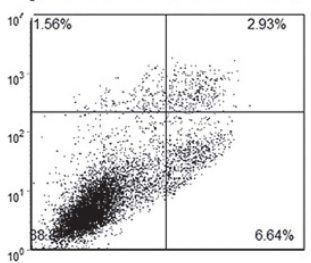

anti-miR-23a/24/27a

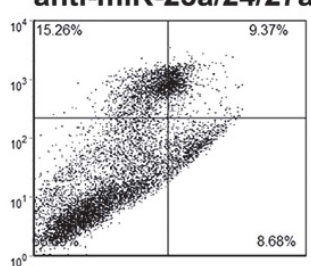

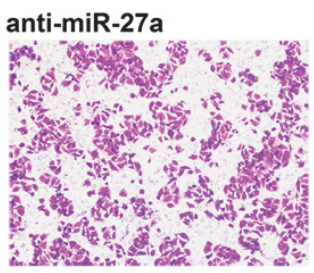

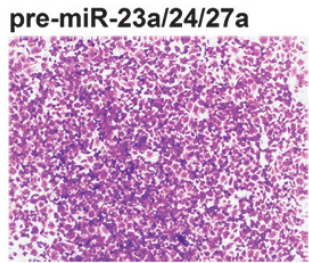

anti-miR-23a/24/27a

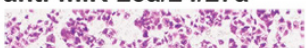

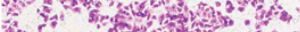
and

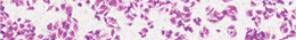
20.

语 h

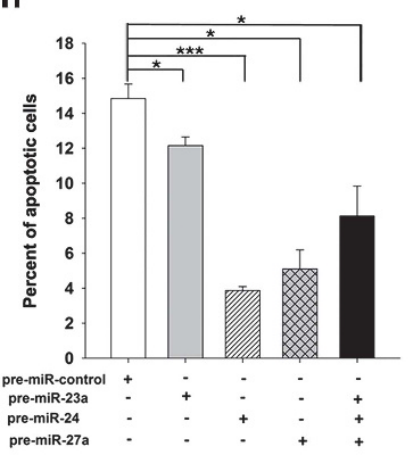

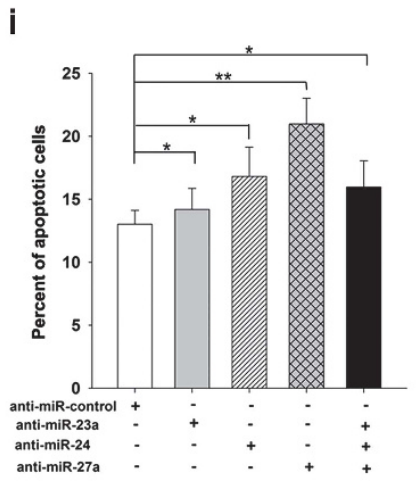

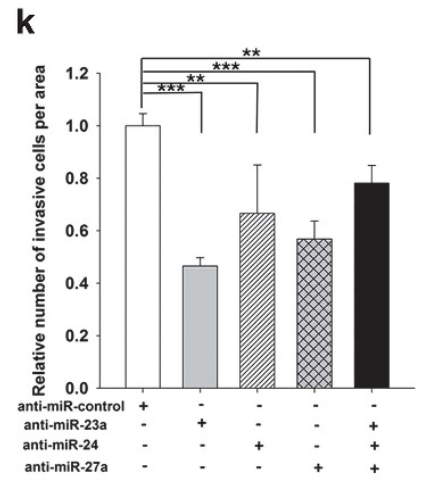


crosstalk between miR-23 27 24 clusters and HIC1 and showed that $\mathrm{HIC} 1$ negatively controls the expression of miR-23 27 24 clusters via specific HIC1-binding motifs.
Thus, HIC1 and miR-23 27 24 clusters form a doublenegative feedback loop that contributes to breast cancer progression.

a

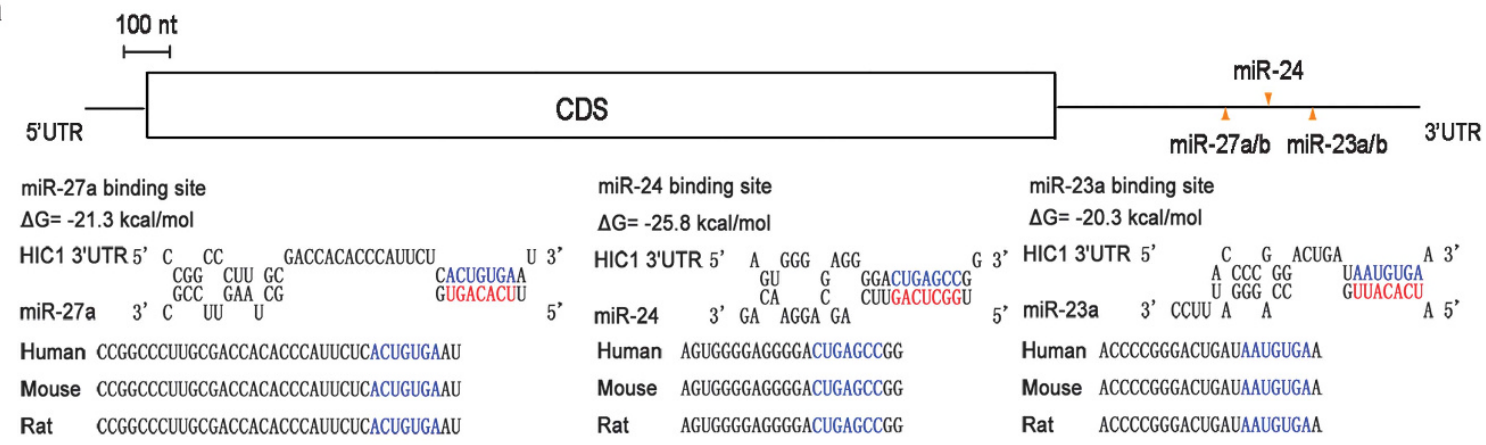

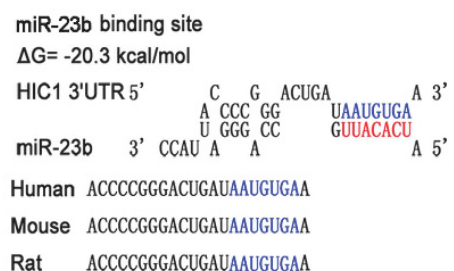

C

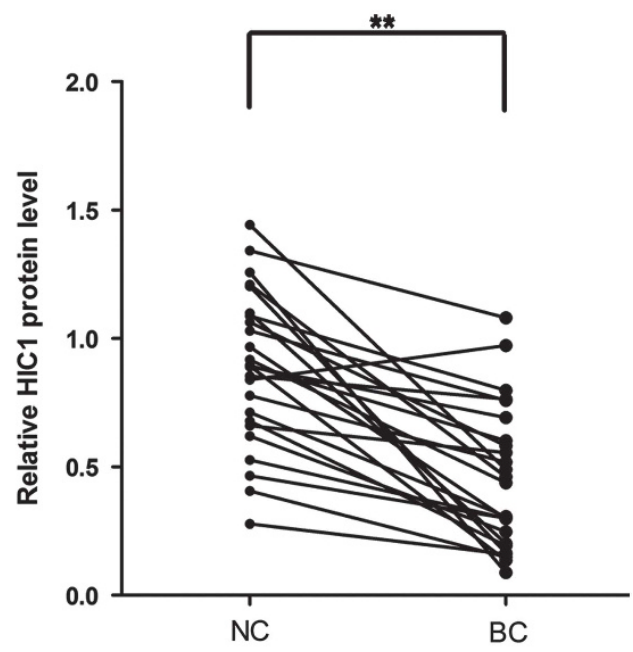

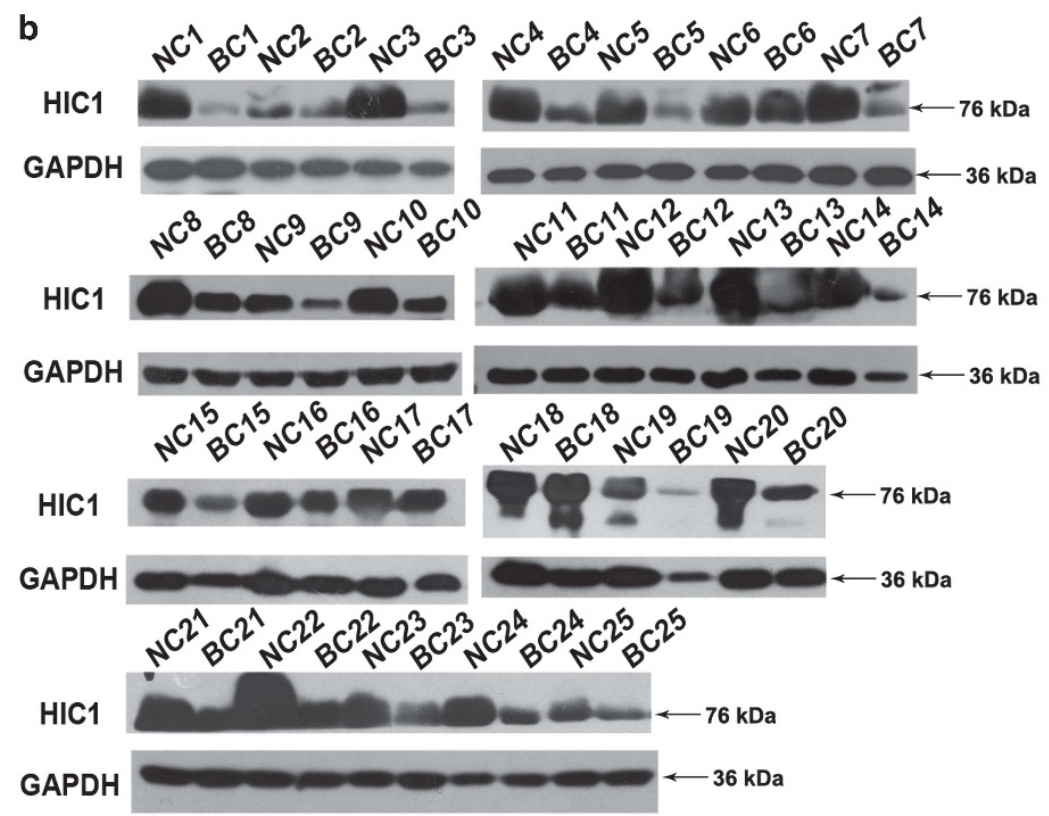

Figure 2 Prediction of $\mathrm{HIC1}$ as a common target of miR-23 $27 \sim 24$ clusters. (a) Schematic depicting the hypothetical duplexes formed by interactions between the binding sites in the HIC1 3'-UTR (top) and miR-27a, miR-24, miR-23a, miR-27b and miR-23b (bottom). The minimum free energy values of the hybridizations are indicated. The seed recognition sites are denoted; all nucleotides in these regions are highly conserved across species, including human, mouse and rat. (b and $\mathbf{c}$ ) Western blotting analysis of the expression levels of HIC1 protein in 25 pairs of $\mathrm{BC}$ and NC samples. (b) representative image; (c) quantitative analysis. ${ }^{*} P<0.01$

Figure 1 Upregulated miR-23a 27a 24-2 and miR-23b 27b 24-1 clusters functioned as oncogenes in breast cancer. (a-e) Quantitative RT-PCR analysis was used to evaluate the expression levels of miR-23a (a), miR-24 (b), miR-27a (c), miR-23b (d) and miR-27b (e) in 25 pairs of breast cancer tissue (BC) and noncancerous tissue (NC) samples. (f, h and i) Analysis of apoptosis in MCF-7 cells treated with pre-miR-control, pre-miR-23a, pre-miR-24, pre-miR-27a or with a mixture of these pre-miRs, or with antimiR-control, anti-miR-23a, anti-miR-24, anti-miR-27a or a mixture of these anti-miRs. The total apoptotic cells were counted as the sum of early apoptotic $\left(\mathrm{PI}^{-} \mathrm{AV}^{+}\right)$and late apoptotic $\left(\mathrm{Pl}^{+} \mathrm{AV}^{+}\right)$cells (f: representative image; $\mathbf{h}$ and $\mathbf{i}$ : quantitative analysis). ( $\mathbf{g}$, j and $\mathbf{k}$ ) Transwell analysis of invaded MCF-7 cells treated with pre-miR-control, pre-miR-23a, pre-miR-24, pre-miR-27a or with a mixture of these pre-miRs, or with anti-miR-control, anti-miR-23a, anti-miR-24, anti-miR-27a or a mixture of these anti-miRs (g: representative image; j and k: quantitative analysis). ${ }^{*} P<0.05 ;{ }^{* *} P<0.01 ;{ }^{* *} P<0.001$ 


\section{Results}

miR-23 $27 \sim 24$ clusters are upregulated in breast cancer tissues. We first determined the expression patterns of miR-23a $\sim 27 a \sim 24-2$ and miR-23b $\sim 27 b \sim 24-1$ clusters in human breast cancer tissues. After measuring the expression levels of miR-23a, miR-27a, miR-24, miR-23b and miR-27b in 25 pairs of breast cancer tissues and corresponding noncancerous tissues, we found that all of these miRNAs were consistently upregulated in breast cancer tissues compared with noncancerous tissues (Figures 1a-e).

miR-23 $27 \sim 24$ clusters inhibit apoptosis and promote invasion in breast cancer cells. We next assessed cell apoptosis and invasion in the human breast cancer cell line MCF-7 after transfecting cells with different types of premiRNA mimics or antisense miRNAs of miR-23 27 24 clusters, individually or simultaneously. As expected, the cellular levels of miR-23a, miR-27a, miR-24, miR-23b and miR-27b increased significantly when the MCF-7 cells were transfected with pre-miRNA mimics, and they decreased dramatically when the MCF-7 cells were treated with antisense miRNAs (Supplementary Figures S1A and S1B). In the cell apoptosis assay, the percentage of apoptotic cells was significantly lower in MCF-7 cells transfected individually or simultaneously with pre-miR-23a, pre-miR-27a and premiR-24 (Figures if and h) and higher in cells transfected individually or simultaneously with anti-miR-23a, antimiR-27a and anti-miR-24 (Figures 1f and i). Likewise, the percentage of apoptotic cells was decreased in MCF-7 cells transfected with pre-miR-23b or pre-miR-27b (Supplementary Figures S2A and S2C). In the Transwell invasion assay, the percentage of invaded cells was significantly higher in MCF-7 cells transfected with pre-miR-23a, pre-miR-27a and premiR-24, respectively or simultaneously (Figures $1 \mathrm{~g}$ and $\mathrm{j}$ ); in contrast, knockdown of miR-23a, miR-27a and miR-24 had an opposite effect on the cell invasion ability of MCF-7 cells (Figures $1 \mathrm{~g}$ and k). Similarly, miR-23b and miR-27b had a positive effect on the invasion ability of MCF-7 cells (Supplementary Figures S2B and S2D). The results suggest that miR-23 27 24 clusters inhibit cell apoptosis and promote cell invasion in breast cancer cells.

Prediction of HIC1 as a common target of miR-23 27 24 clusters. To assess the cooperative effects of the miR$-23 \sim 27 \sim 24$ clusters, the common target genes of miR-23a/b (miR-23a and miR-23b share identical seed sequences and therefore common targets), miR-27a/b and miR-24 were predicted using three computational algorithms TargetScan, ${ }^{13}$ miRanda $^{14}$ and PicTar. ${ }^{15}$ HIC1, a tumorsuppressor gene that is downregulated in many human cancers, ${ }^{16}$ was identified as an ideal candidate. The predicted interactions between miR-23 27 24 clusters and HIC1 3'-UTR are illustrated in Figure 2a.

We next investigated whether the expression of miR$-23 \sim 27 \sim 24$ clusters was inversely correlated with the expression of HIC1 in breast cancer. By measuring the levels of $\mathrm{HIC} 1$ protein in the same 25 pairs of breast cancer tissues and corresponding noncancerous tissues, we found that HIC1 protein levels were consistently downregulated in the cancer tissues (Figures $2 b$ and c). However, it should be noted that the expression levels of HIC1 protein appeared very heterogeneous among normal tissues. The inconsistent expression of HIC1 protein in normal tissues might be due to the difference among age and other clinical features of the patients and requires further validation in a larger cohort.

Validation of HIC1 as a common target of miR-23 27 24 clusters. To further validate the correlation between miR-23 27 24 cluster expression and HIC1 expression, we assessed the levels of HIC1 protein in MCF-7 cells after transfection with pre-miRNA mimics or antisense miRNAs, individually or simultaneously. The expression of HIC1 protein in MCF-7 cells was significantly inhibited by the introduction of miR-23a, miR-27a and miR-24, whereas antisense miRNAs of miR-23a, miR-27a and miR-24 significantly increased HIC1 protein levels in the cells (Figures 3a and b). Likewise, the expression of HIC1 protein was suppressed by the introduction of miR-23b and miR-27b in MCF-7 cells (Figures $3 a$ and $b$ ). To test the robustness of the effect, we repeated the above experiments in an additional breast cancer cell line, MBA-MD-231, and observed consistent results (Supplementary Figures S1C, S1D, S3A-S3C). To determine the regulatory level at which the miR-23 27 24 cluster influences $\mathrm{HIC1}_{1}$ expression, we repeated the above experiments and examined the expression of HIC1 mRNA after transfection. Overexpression or knockdown of $\mathrm{miR}-23 \mathrm{a} / \mathrm{b}, \mathrm{miR}-27 \mathrm{a} / \mathrm{b}$ and $\mathrm{miR}-24$ did not affect HIC1 mRNA levels in MCF-7 cells (Supplementary Figures $3 \mathrm{D}$ and $3 \mathrm{E})$.

To confirm that the miR-23 27 24 cluster directly targets the presumed binding sites in the $3^{\prime}$-UTR of HIC1 mRNA and negatively regulates $\mathrm{HIC1}$ expression, a luciferase reporter assay was performed. The full-length 3 '-UTR of HIC1 containing the three presumed binding sites for the miRNAs was placed downstream of the firefly luciferase gene in a reporter plasmid. As anticipated, luciferase activity was remarkably reduced in cells co-transfected with the luciferase reporter plasmid and pre-miRNA mimics of miR-23a, miR-27a, miR-24, miR-23b and miR-27b individually or simultaneously (Figure 3c). Furthermore, we introduced point mutations into the corresponding complementary sites in the $3^{\prime}$-UTR of HIC1 to eliminate the predicted binding sites. This altered luciferase reporter was unaffected by the overexpression of miR-23a, miR-27a, miR-24, miR-23b and miR-27b (Figure 3c), suggesting that the binding sites strongly contribute to an miRNA: mRNA interaction that mediates the post-transcriptional repression of HIC1 expression.

HIC1 attenuates the anti-apoptotic and pro-invasive effects of the miR-23a 27a 24-2 cluster in breast cancer cells. HIC1 is known to induce apoptosis and suppress cell invasion. ${ }^{16,17}$ To test whether miR$-23 a \sim 27 a \sim 24-2$ may suppress HIC1 expression to affect cell apoptosis and invasion, we transfected MCF-7 cells with both mixture of pre-miR-23a, pre-miR-27a, pre-miR-24 and a plasmid designed to specially express the full-length ORF of HIC1 without the miR-23a 27a 24-2-responsive 3'-UTR. Efficient overexpression of HIC1 in MCF-7 cells was achieved (Supplementary Figures S4A and S4C). In agreement with 
a
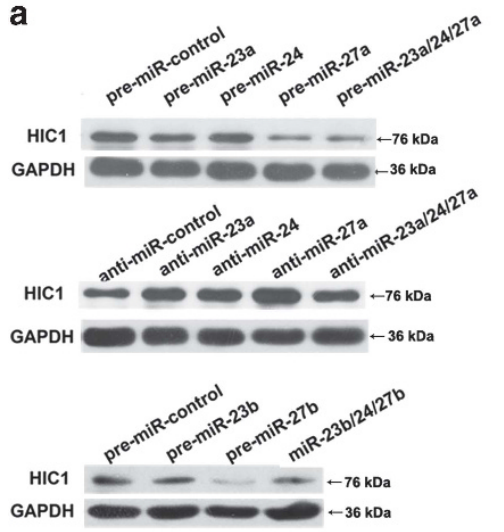

b

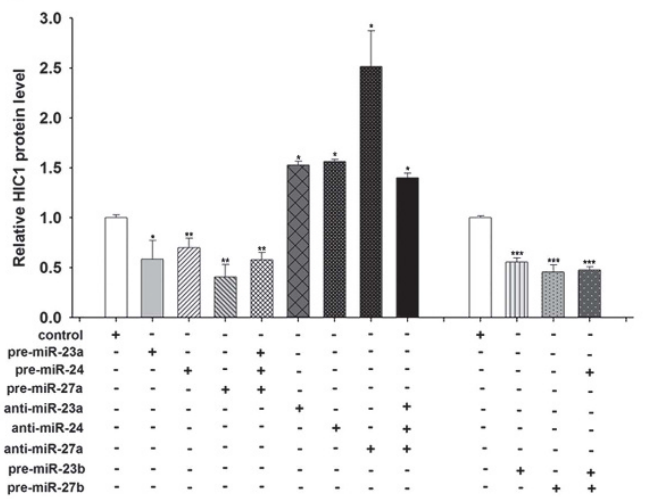

C

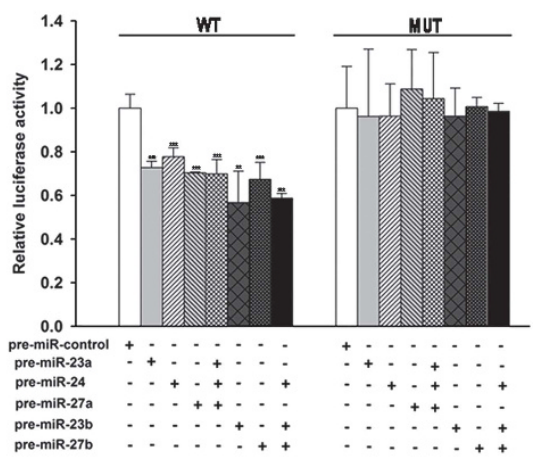

d

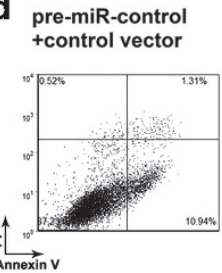

pre-miR-23a/24/27a +control vector

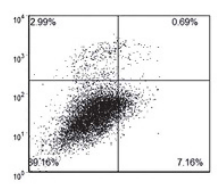

e

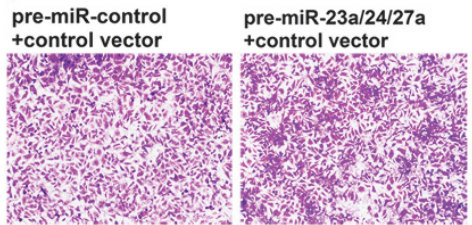

pre-miR-control +HIC1 vector

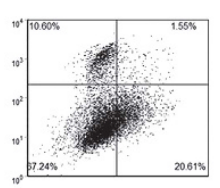

pre-miR-control pre-miR-cont
+ HIC1 vector + HIC1 vector

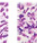

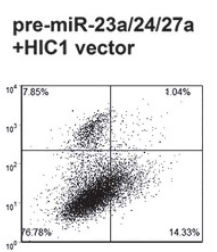

f

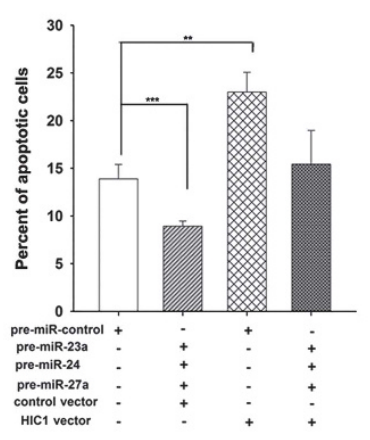

g

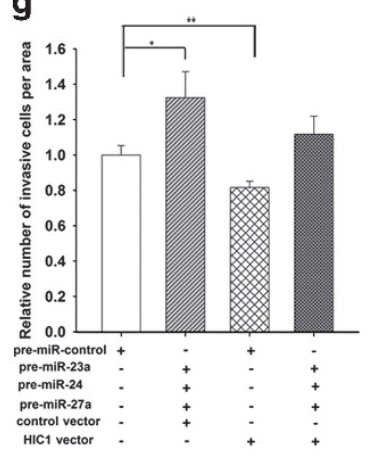

Figure 3 HIC1 is a direct target of miR-23 27 24 clusters. (a and $\mathbf{b})$ Western blot analysis of the expression levels of HIC1 protein in MCF-7 cells treated with pre-miRcontrol, pre-miR-23a/b, pre-miR-24, pre-miR-27a/b or a mixture of these pre-miRs, or with anti-miR-control, anti-miR-23a, anti-miR-24, anti-miR-27a or a mixture of these antimiRs; a: representative image; (b) quantitative analysis. (c) Firefly luciferase reporters containing wild-type (WT) or mutant (MUT) miR-23a/b, miR-24 and miR-27a/b binding sites in the HIC1 3'-UTR were co-transfected into 293T cells together with pre-miR-control, pre-miR-23a/b, pre-miR-24, pre-miR-27a/b or a mixture of these pre-miRs. Twenty-four hours post transfection, the cells were assayed using a luciferase assay kit. (d and f) Analysis of apoptosis in MCF-7 cells treated with pre-miR-control plus control vector, premiR-23a/24/27a mixture plus control vector, pre-miR-control plus HIC1 vector, or pre-miR-23a/24/27a mixture plus HIC1 vector. The total apoptotic cells were counted as the sum of early apoptotic $\left(\mathrm{Pl}^{-} \mathrm{AV}^{+}\right)$and late apoptotic $\left(\mathrm{PI}^{+} \mathrm{AV}{ }^{+}\right)$cells (d: representative image; $\mathrm{f}$ : quantitative analysis). (e and $\left.\mathbf{g}\right)$ Transwell analysis of invaded MCF-7 cells treated with with pre-miR-control plus control vector, pre-miR-23a/24/27a mixture plus control vector, pre-miR-control plus HIC1 vector, or pre-miR-23a/24/27a mixture plus HIC1 vector (e: representative image; g: quantitative analysis). ${ }^{\star} P<0.05 ;{ }^{* \star} P<0.01 ;{ }^{* \star \star} P<0.001$

previous findings, ${ }^{16,17}$ overexpression of $\mathrm{HIC} 1$ in MCF-7 cells indeed caused increased cell apoptosis and decreased cell invasion (Figures $3 \mathrm{~d}-\mathrm{g}$ ), indicating that $\mathrm{HIC1}$ and miR$-23 a \sim 27 a \sim 24-2$ cluster have the opposite effect on cell apoptosis and invasion. More importantly, apoptosis and invasion assays revealed that ectopic expression of miR-23a 27a 24-2-resistant HIC1 dramatically attenuated the inhibitory effect of the miR-23a $\sim 27 \mathrm{a} \sim 24-2$ cluster on cell apoptosis and stimulatory effect on cell invasion (Figures 3dg). Because miR-23 27 24-resistant HIC1 could rescue the suppression of $\mathrm{HIC1}$ caused by miR-23 27 24 clusters and attenuate the anti-apoptotic and pro-invasive effects of the miR-23 27 24 clusters, targeting HIC1 is at least one mechanism through which miR-23 27 24 clusters exert the oncogenic role.

miR-23 $27 \sim 24$ and HIC1 forms a double-negative feedback loop. The reason for the upregulation of miR$-23 \sim 27 \sim 24$ cluster expression during breast tumorigenesis is largely unknown. We therefore predicted putative transcription factor binding sites in the promoter region of miR$-23 a \sim 27 a \sim 24-2$ and miR-23b $\sim 27 b \sim 24-1$ clusters. Interestingly, genomic analysis identified three HIC1-binding motifs consisting of a $5^{\prime}-(\mathrm{C} / \mathrm{G}) \mathrm{NG}(\mathrm{C} / \mathrm{G}) \mathrm{GGGCA}(\mathrm{C} / \mathrm{A}) \mathrm{CC}-3^{\prime}$ sequence $^{18}$ within the promoter region of the miR$-23 a \sim 27 a \sim 24-2$ cluster and one motif within the promoter region of the C9orf3 gene (miR-23b $27 \mathrm{~b} \sim 24-1$ cluster is located in the sixteenth intron of C9orf3 gene) (Figure 4a). Hence, we investigated whether HIC1, as a sequencespecific transcriptional repressor, can recognize and bind to the HIC1-responsive elements and negatively control the expression of miR-23a 27a 24-2 and miR-23b 27b 24-1 clusters. To address whether HIC1 protein is recruited to the specific motifs in miR-23a 27a $24-2$ and miR$-23 b \sim 27 b \sim 24-1$ promoters, we performed chromatin immunoprecipitation (ChIP) assays in MCF-7 cells. As anticipated, ChIP assays using antibody against HIC1 showed robust PCR product enrichment indicative of $\mathrm{HIC1}$ binding at sites 1 
a

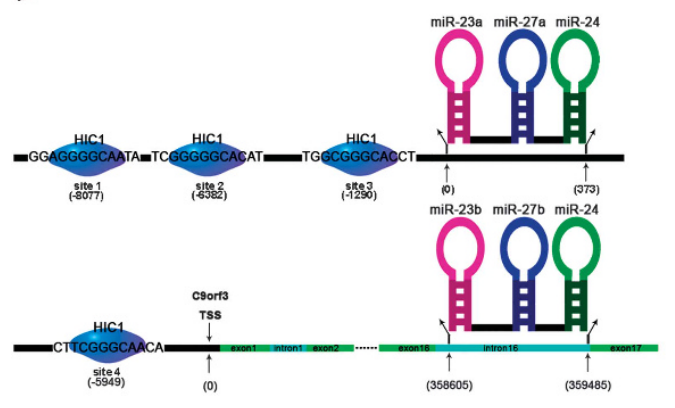

b

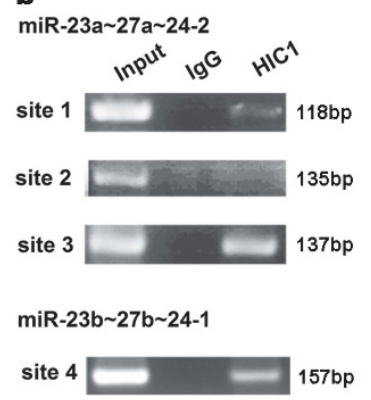

C

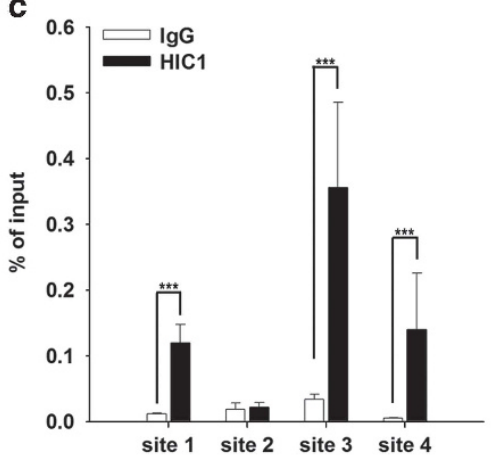

d
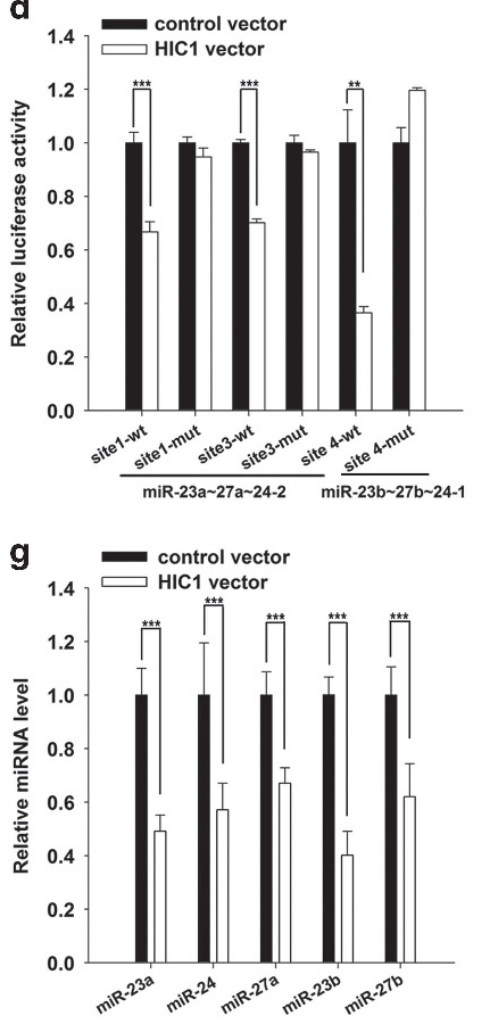

e

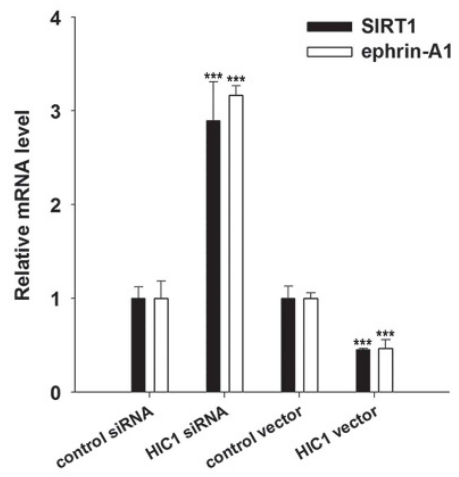

h

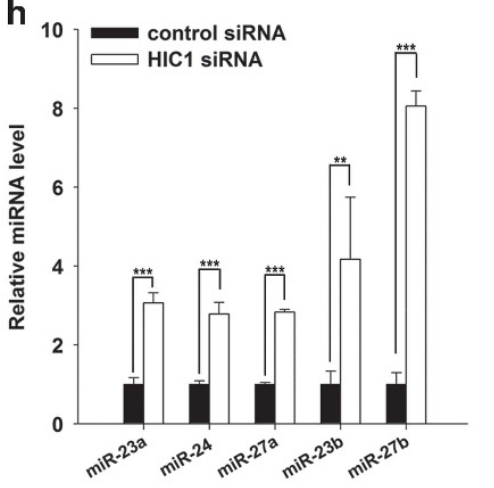

i

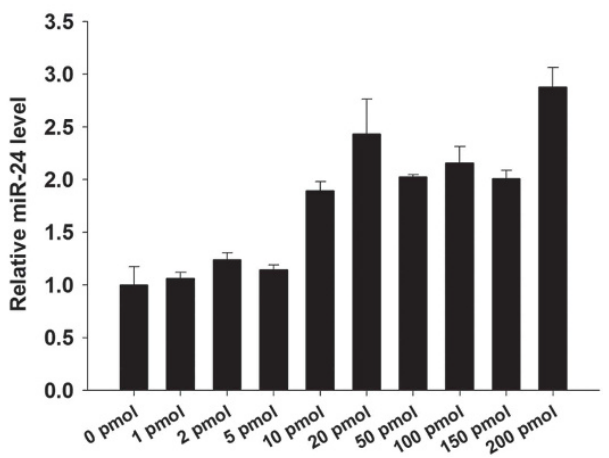

Figure 4 HIC1 directly inhibits miR-23 27 24 clusters. (a) Schematic illustrating the three putative HIC1-binding motifs (sites 1, 2 and 3) in the miR-23a 27a 24-2 promoter and one putative HIC1-binding motif (site 4 ) in the miR-23b 27b 24-1 promoter. (b and c) Direct binding of HIC1 to promoter regions of miR-23 27 24 clusters indicated by PCR-based ChIP assays. Robust PCR product enrichment indicating HIC1 binding is shown in the anti-HIC1 lane. Negative control amplification was carried out on rabbit lgG-immunoprecipitated chromatin (IgG lane). Positive control amplification was carried out on input chromatin before immunoprecipitation (Input lane). Binding of HIC1 to sites 1, 3 and 4, but not to site 2, was confirmed by semi-quantitative PCR followed by gel electrophoresis (b) and quantitative PCR (c) using primers specific for the four sites. (d) Luciferase reporter assays confirming the suppression of miR-23 27 24 promoters by HIC1 through the three potential HIC1-binding motifs (sites 1, 3 and 4). Expression of firefly luciferase was regulated by miR-23 27 24 promoter sequences containing either wild-type (site 1-wt, site 3-wt and site 4-wt) or mutated (site 1-mut, site 3-mut and site 4mut) HIC1-binding motifs. Ectopic expression of $\mathrm{HIC1}$ downregulates luciferase expression from plasmids that contain wild-type but not mutant HIC1-binding motifs in the promoter. Firefly luciferase signals were normalized based on $\beta$-gal expression by a simultaneously transfected $\beta$-gal expression plasmid. (e and f) Quantitative RT-PCR (e) and western blot (f) analysis of the mRNA and protein levels of SIRT1 and ephrin-A1 in MCF-7 cells after knockdown or overexpression of HIC1. (g) Quantitative RT-PCR showing that HIC1 knockdown by siRNA upregulates the expression of miR-23a, miR-24, miR-27a, miR-23b and miR-27b in MCF-7 cells. (h) Quantitative RT-PCR showing that ectopic HIC1 expression downregulates the expression of miR-23a, miR-24, miR-27a, miR-23b and miR-27b in MCF-7 cells. (i) Quantitative RT-PCR analysis of the miR-24 levels in MCF-7 cells after treatment with increased doses of HIC1 siRNA. ${ }^{* \star} P<0.01 ;{ }^{* \star \star} P<0.001$

and 3 in the miR-23a $\sim 27 a \sim 24-2$ promoter and at site 4 in the miR-23b 27b 24-1 promoter (Figures $4 b$ and c). Next, we cloned the sequences of HIC-binding sites 1, 3 and 4 into an upstream region of a firefly luciferase reporter gene and transfected the resulting plasmids into 293T cells. Luciferase reporter assays revealed that ectopic expression of HIC1 inhibited the transcription of firefly luciferase in plasmids in which $\mathrm{HIC} 1$ binding site 1,3 or 4 sequences had been inserted into the promoter region; however, when the binding sequences of sites 1, 3 and 4 were mutated, firefly luciferase activity was unaffected by HIC1 overexpression (Figure 4d). Furthermore, we overexpressed and knocked down HIC1 in 
MCF-7 cells and measured the response of miR-23a $\sim 27 \mathrm{a} \sim 24-2$ and miR-23b $\sim 27 \mathrm{~b} \sim 24-1$ clusters by quantitative RT-PCR. Efficient knockdown of HIC1 in MCF-7 cells was achieved, as shown in Supplementary Figures S4A and S4B. For the canonical target gene of HIC1, SIRT1 and ephrin-A1, ${ }^{17,20}$ silencing of $\mathrm{HIC1}$ caused significant
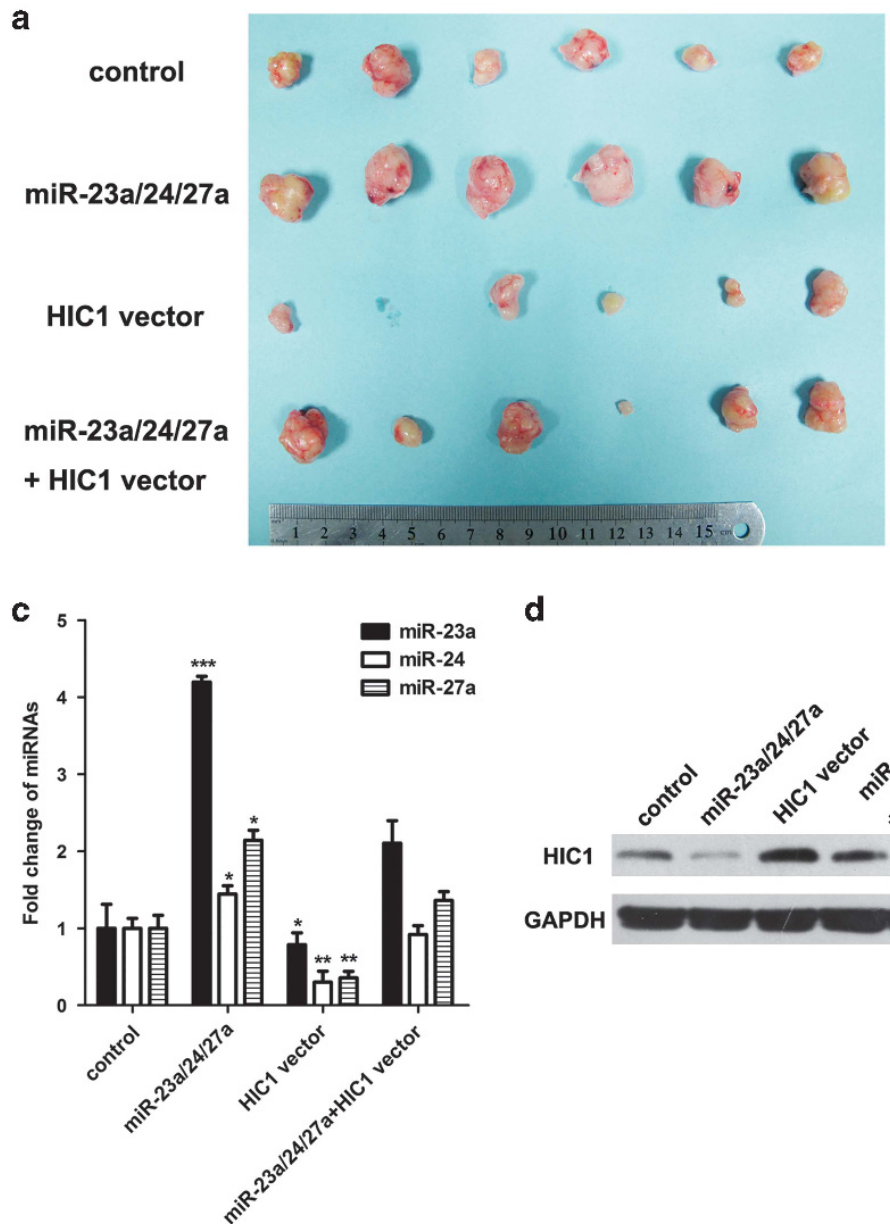

d

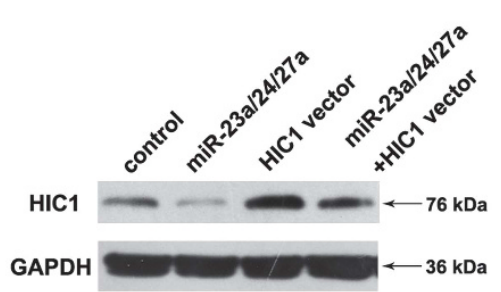

b

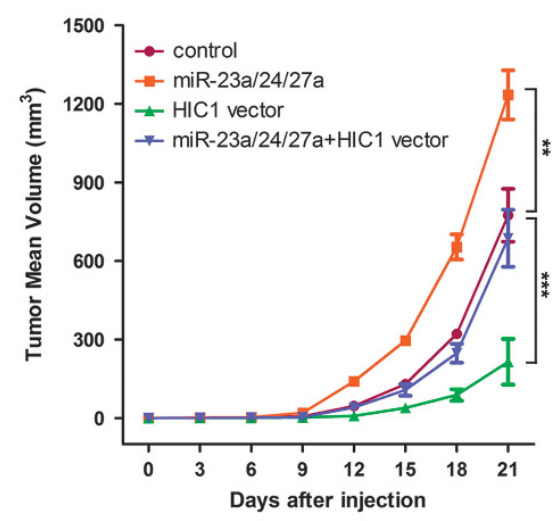

e

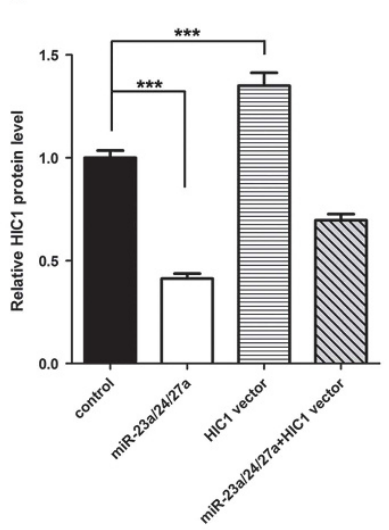

$\mathbf{f}$
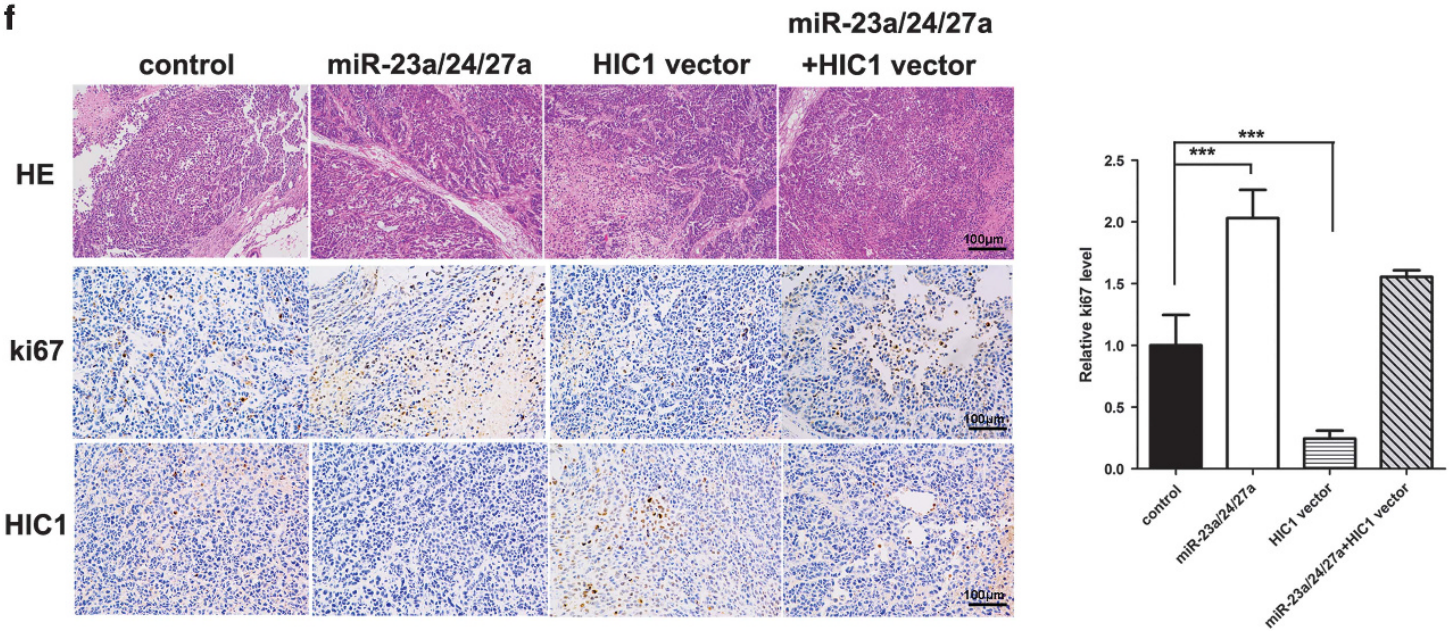

Figure 5 Effects of $\mathrm{HIC1}$ and miR-23a 27a 24-2 cluster overexpression on the growth of breast cancer cell xenografts in mice. (a) Representative images of tumors from mice implanted with control MCF-7 cells, miR-23a/24/27a-overexpressing MCF-7 cells, HIC1 vector-overexpressing MCF-7 cells or miR-23a/24/27a and HIC1 co-overexpressing MCF-7 cells. MCF-7 cells $\left(2 \times 10^{6}\right.$ cells per $\left.0.1 \mathrm{ml}\right)$ with different treatments were implanted subcutaneously into 6 -week-old SCID mice (six mice per group). (b) The time course of tumor growth in implanted mice. Tumor volume was measured every 3 days for 21 days after inoculation. (c) Quantitative RT-PCR analysis of miR-23a, miR-24 and miR-27a levels in tumors from the implanted mice. (d and e) Western blotting analysis of HIC1 protein levels in tumors from the implanted mice; (d) representative image; (e) quantitative analysis. (f) Tumors from the implanted mice were subjected to H\&E staining and immunohistochemical staining for Ki-67 and HIC1. The right histograms represent quantitative analyses of Ki-67-positive signals in the tumor. All data are shown as the means \pm S.E. obtained from three separate experiments. ${ }^{\star} P<0.05 ;{ }^{* \star} P<0.01 ;{ }^{* \star *} P<0.001$ 
upregulation of the mRNA and protein levels of SIRT1 and ephrin-A1 in MCF-7 cells, whereas overexpression of HIC1 showed an opposite effect on their expression (Figures $4 \mathrm{e}$ and f). Likewise, ectopic expression of HIC1 in MCF-7 cells resulted in a 2- to 4-fold decrease in miR-23a, miR-27a, miR-24, miR-23b and miR-27b expression compared with cells transfected with a control vector, whereas HIC1 knockdown by siRNA resulted in a two- to three-fold increase in miR-23a, miR-27a, miR-24, miR-23b and miR-27b expression levels (Figures $4 g$ and $h$ ). Similar alteration in the levels of the precursors of miR-23 27 24 clusters was observed (Supplementary Figures S5A and S5B), suggesting that the alteration of miR-23 27 24 clusters was likely due to the transcriptional changes. For miR-23b 27b 24-1 cluster located within the sixteenth intron of C9orf3 gene, a change in the expression of miR-23b $\sim 27 b \sim 24-1$ was accompanied by a concordant change in the expression of the C9orf3 mRNA and the sixteenth intron (Supplementary Figures S5C and S5D). Moreover, we performed an siRNA titration experiment to validate that the upregulation of $\mathrm{miR}$ $-23 \sim 27 \sim 24$ clusters was specifically caused by HIC1. HIC1 siRNA was added in a dose-dependent manner to MCF-7 cells to neutralize the suppression of $\mathrm{HIC1}$ on miR-23 27 24 clusters. As expected, HIC1-guided control of miR-24 expression was gradually relieved by increasing HIC1 siRNA input (Figure 4i). Taken together, these results demonstrate that $\mathrm{HIC1}$ negatively regulates the transcription of miR-23a $27 a \sim 24-2$ and miR$-23 b \sim 27 b \sim 24-1$ clusters via specific HIC1-binding motifs in the promoter regions.

The influence of HIC1-miR-23a 27a 24-2 doublenegative feedback loop on the growth of breast cancer cells in vivo. We next evaluated the effects of $\mathrm{HIC1}$ miR-23a $\sim 27 a \sim 24-2$ double-negative feedback loop on the growth of breast cancer cell xenografts in mice. The MCF-7 cells were infected with three lentiviral expression vectors to co-express miR-23a, miR-24 and miR-27a (miR-23a/24/27a). Efficient co-expression of miR-23a/24/27a and inhibition of HIC1 in MCF-7 cells by transfection with these lentiviral vectors is shown in Supplementary Figure S6. The MCF-7 cells were also transfected with a plasmid for overexpression of $\mathrm{HIC1}$ or co-transfected with the miR-23a/24/27a overexpression lentivirus plus HIC1 overexpression plasmid; then, the cells were implanted subcutaneously into 6-weekold nude mice, and tumor growth was evaluated on day 21 after cell implantation. A significant increase in the sizes and weights of the tumors was observed in the miR-23a/24/27aoverexpressing group compared with the control group, whereas the tumors from the HIC1-overexpressing group grew dramatically slower (Figures $5 \mathrm{a}$ and b). In addition, the group with both miR-23a/24/27a and HIC1 overexpression exhibited a significantly lower tumor growth compared with the group with miR-23a/24/27a overexpression alone (Figures $5 \mathrm{a}$ and $\mathrm{b}$ ), suggesting that $\mathrm{HIC1}$ overexpression can attenuate the promotive effect of miR-23a/24/27a on tumor growth. Subsequently, total RNA and protein were extracted from each xenograft and used to evaluate the expression levels of miR-23a/24/27a and HIC1. After 21 days of xenograft growth in vivo, tumors from the miR-23a/24/27aoverexpressing group showed a significant increase in the expression of miR-23a/24/27a and displayed reduced HIC1 protein levels compared with tumors from the control group (Figures 5c-e). Tumors with both miR-23a/24/27a and HIC1 overexpression exhibited significantly higher levels of HIC1 compared with tumors with miR-23a/24/27a overexpression (Figures 5d and e), suggesting that miR-23a/24/27a-resistant HIC1 is sufficient to rescue the suppression of HIC1 caused by miR-23a/24/27a. Consistent with this, immunohistochemical studies also revealed the presence of lower levels of HIC1 in the group implanted with miR-23a/24/27a-overexpressing cells (Figure 5f). In addition, hematoxylin and eosin (H\&E) staining of the xenograft tissues also showed more cell invasion and less cell apoptosis in tumors of the miR-23a/24/27a-overexpressing group than in tumors of the control group (Figure 5f). Xenografts with both miR-23a/ 24/27a and HIC1 overexpression exhibited reduced cell mitosis and increased cell apoptosis compared to xenografts with $\mathrm{miR}-23 \mathrm{a} / 24 / 27 \mathrm{a}$ overexpression (Figure 5f), suggesting that HIC1 overexpression could attenuate the pro-invasive and anti-apoptotic effect of miR-23a/24/27a. Finally, the proliferative activity of the tumor cells was assessed via immunocytochemistry using the monoclonal antibody Ki-67. The percentage of Ki-67-positive tumor cells was increased in tumors from the miR-23a/24/27a-overexpressing group, and miR-23a/24/27a-resistant HIC1 is sufficient to reverse the stimulated effect of $\mathrm{miR}-23 \mathrm{a} / 24 / 27 \mathrm{a}$ on cell proliferation (Figure 5f). These results validate the biological role of HIC1-miR-23a 27a 24-2 double-negative feedback loop in breast cancer progression.

miR-23 27 24 clusters and HIC1 act in a doublenegative feedback loop and may constitute a bistable system. The above results indicate that miR-23 27 24 clusters and HIC1 participate in a double-negative (overall positive) feedback loop by mutual repression of each other's expression (Figure 6a). Through a double-negative feedback loop, it is possible to generate two mutually switchable steady states (bistability) within a cancer cell population. ${ }^{20,21}$ The cells in the 'high HIC1/low miR-23 27 24' state can abruptly shift to the 'low HIC1/high miR-23 27 24' state without experiencing an intermediate state in response to sufficient stimulus. Thus, single-cell switching can generate a stable subpopulation of invasive cells that accelerate breast tumorigenesis in the absence of genetic changes. ${ }^{21,22} \mathrm{We}$ created a mathematical model to characterize the regulatory interaction of miR-23 27 24 and HIC1 by describing the steady-state level of miR-24 and $\mathrm{HIC} 1$ with respect to various synthesis rates (Supplementary Methods). Mathematical analysis revealed that both upregulation of miR-24 expression (Figures $6 \mathrm{~b}$ and $\mathrm{c}$ ) and downregulation of $\mathrm{HIC1}$ expression (Figures $6 \mathrm{~d}$ and e) could drive switching of the steady-state level of miR-24 and HIC1 in the system. The model predicted the existence of a threshold $\left(S_{\text {on }}\right.$ in Figures $6 b$ and $c)$, above which the level of HIC1 could abruptly decrease, accompanied by simultaneous increase in the miR-24 level. Once the level of HIC1 decreased upon activation of the switch, HIC1 expression was difficult to 
a

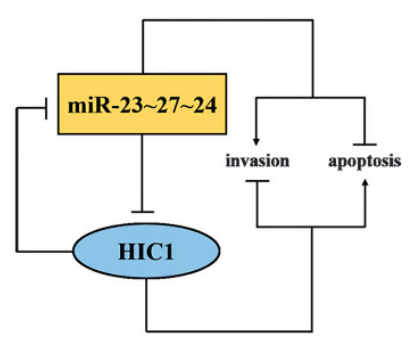

b

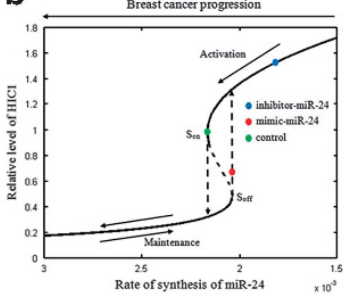

d

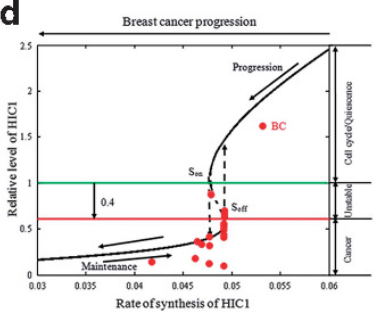

C

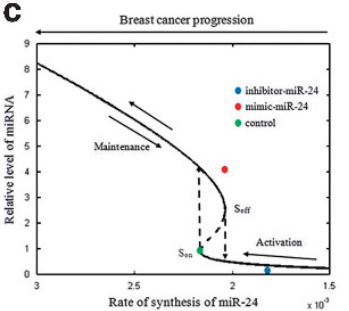

e

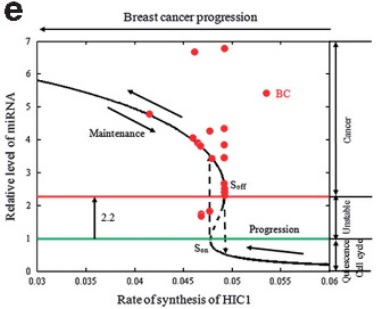

f

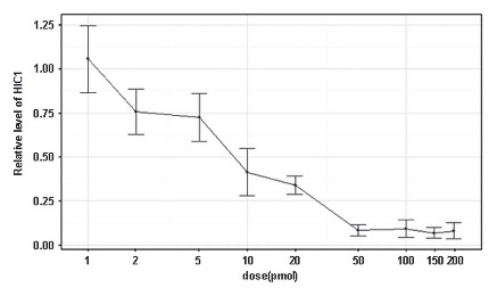

g

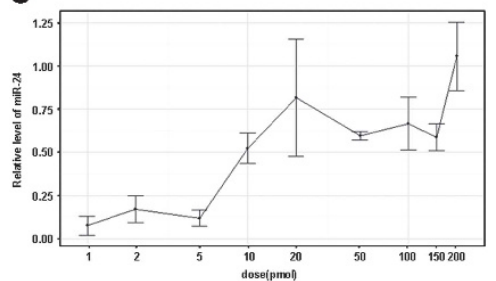

Figure 6 Modeling the double-negative feedback loop comprised HIC1 and miR-23 27 24 clusters. (a) Schematic illustration of the double-negative (overall positive) feedback loop. miR-23 $27 \sim 24$ clusters and HIC1 form a double-negative feedback loop and affect independent pathways that at some point converge onto apoptosis and cell invasion. Pointed arrows and blunted arrows indicate activation and repression, respectively. (b and $\mathbf{c}$ ) Steady-state bifurcation diagram of the levels of HIC1 (b) and miR-24 (c) for different rates of synthesis of miR-24 $\left(\mathrm{k}_{3}=1.5 \sim 3 \times 10^{-3} \mu \mathrm{M} / \mathrm{h}\right)$. The experimental measurement of the relative levels of miR-24 after transient transfection of pre-miR-24 and anti-miR-24 (experimental data are present in Supplementary Figures S1A and S1B) is converted to the functional one and presented as mean. $S_{\text {on }}$ and $S_{\text {off }}$ are two threshold beyond or below which there is a switch of HIC1 and miR-24 levels. (d and e) Steady-state bifurcation diagram of the levels of HIC1 (d) and miR-24 (e) for different rates of synthesis of $\mathrm{HIC1}\left(\mathrm{k}_{1}=0.03-0.06 \mu \mathrm{M} / \mathrm{h}\right)$. BC represents breast cancer tissue (22 out of $25 \mathrm{intal}$, as presented in Figure $1 \mathrm{c}$ and Figure $2 \mathrm{c}$ ). The relative levels of HIC1 and miR-24 were calculated by comparing with theoretical basal HIC1 and miR-24 level concentration (see Supplementary Methods for detail) or dividing the experimentally measured expression of $\mathrm{HIC} 1$ and miR-24 in BC samples by those in NC samples. Each sample was plotted in pair and had the same synthesis rate. (f and $\mathbf{g})$ Relative levels of HIC1 (f) and miR-24 (g) after treatment with a series of HIC1 siRNA titration (0, 1, 2, 5, 10, 20, 50, 100 and 200 pmol, three replicates for each concentration). GAPDH and U6 were served as controls for $\mathrm{HIC} 1$ or miR-24, respectively. Relative fold change was normalized to the average of $0 \mathrm{pmol}$

recover even though the synthesis rate of miR-24 fell below the initial activation threshold $\left(S_{\text {on }}\right.$ in Figures $6 b$ and $c$ ). Maintenance of this state could persist until the rate of synthesis of miR-24 fell below another threshold $\left(S_{\text {off }}\right.$ in Figures $6 b$ and $c)$.

We next investigated the impact of decreasing the synthesis rate of $\mathrm{HIC1}$, as an analog to epigenetic downregulation of HIC1, on the steady-state level of the system. Similarly, the model predicted both switch and hysteresis under these conditions. Perhaps due to biological variability and difference between single cell and population measurement, the predictions of the model did not perfectly match the experimental results. However, the trend and the switching of the steadystate level of the system were noticeable (Figures $6 \mathrm{~d}$ and e). Because miR-24 is an oncomiR and HIC1 is a tumor suppressor, the abrupt increase in miR-24 and the decrease in HIC1 could be viewed as a feature of breast cancer progression. The model was able to distinguish between cancer and the normal cell cycle state based on the switching of the steady-state levels of miR-24 and HIC1. Most samples (18 out of 22, three outlier removed) were diagnosed as being in the cancer state when the $\mathrm{HIC} 1$ level fell below $60 \%$ and the miR-24 level doubled. We also performed HIC1 siRNA titration assay, in analog to epigenetic downregulation, to test the existence of the switch (Figures $6 f$ and g). Indeed, the relative level of $\mathrm{HIC} 1$ and miR-24 did not react linearly to the gradient of transfection, which suggests the existence of regulatory interaction between $\mathrm{HIC} 1$ and miR-23 27 24. Furthermore, we found a rapid alteration of $\mathrm{HIC1}$ ( 0.4-fold decrease) and miR-24 ( 2-fold increase) levels when increasing siRNA gradient from 5 to $10 \mathrm{pmol}$ (bistability may occur in this interval). Thus, the model theoretically validated the experimental results and suggested that a double-negative feedback loop might generate bistability and accelerate the cancer progression.

\section{Discussion}

Recently, an important role for miRNA in the genesis and progression of breast cancer has emerged. ${ }^{5}$ In this study, we showed that miR-23 27 24 clusters can directly target $\mathrm{HIC} 1$, while $\mathrm{HIC} 1$ can, in turn, be recruited to miR-23 27 24 promoters to repress the expression of miR-23 27 24 clusters. Thus, miR-23 27 24 clusters and HIC1 form a double-negative feedback loop that may amplify miR$-23 \sim 27 \sim 24$ clusters and minimize HIC1 expression in breast cancer cells. This feedback regulation may explain the widespread downregulation of $\mathrm{HIC1}$ and the overexpression of miR-23 27 24 clusters in human cancers. How could such a feedback loop participate in or accelerate breast carcinogenesis? Because double-negative feedback is equal to positive feedback and is known for its ability to amplify a response into a self-sustained mode that is independent of the original stimuli, the feedback loop composed of $\mathrm{HIC} 1$ and miR-23 27 24 clusters may allow breast cancer cells to become more autonomous, for example, to reproduce more rapidly and to metastasize to new microenvironments.

To date, most miRNA research has focused on the individual effects of specific miRNAs. It is imperative to study the cooperative effects of multiple miRNAs. One goal of this 
study is to determine whether members of miR-23 27 24 clusters possess overlapping targets and work in combination to accomplish their functions. Here, HIC1 was identified as a common target gene of miR-23 27 24 clusters. Because very few demonstrations of clusters of miRNAs that target the same genes have been provided to date, our results provide novel evidence that individual miRNAs within a cluster that display co-expression but lack sequence homology can simultaneously and cooperatively repress a given target gene. Cooperation among the clustered miRNAs thus provides an interesting area of study that may change our perception of how miRNAs mediate gene expression in regulatory networks.

Our findings may also open new avenues for breast cancer therapies. In principle, restoration of $\mathrm{HIC1}$ expression in cancer cells would inhibit the expression of oncogenic miR$-23 \sim 27 \sim 24$ clusters, which in turn would relieve the suppression of HIC1 expression, thereby accelerating the accumulation of $\mathrm{HIC} 1$ in cancer cells and slowing tumor growth. In agreement with this theory, restoration of HIC1 attenuated the anti-apoptotic and pro-invasive effect of miR$-23 \sim 27 \sim 24$ clusters in breast cancer cells and blocked tumor growth in nude mice. Thus, $\mathrm{HIC1}$ may be an attractive target for future breast cancer therapy. Considerable further research should be performed to develop feasible strategies to restore HIC1 expression in vivo.

Taken together, this study describes a double-negative feedback loop comprised of HIC1 and miR-23 27 24 clusters in breast cancer. This finding is consistent with the increasingly prevalent view that miRNAs form regulatory motifs with protein regulators to confer robustness to biological processes and that their disorder can expose cells to an elevated risk of dysfunction.

\begin{abstract}
Materials and Methods
Cells and human tissues. The human breast cancer cell lines MCF-7 and MDA-MB-231 were purchased from the Shanghai Institute of Cell Biology, Chinese Academy of Sciences (Shanghai, China). MCF-7 was cultured in DMEM, and MDAMB-231 cells were cultured in L15 medium; the culture media were supplemented with $10 \%$ fetal bovine serum (Gibco, Carlsbad, CA, USA), and the cells were incubated in $5 \% \mathrm{CO}_{2}$ at $37{ }^{\circ} \mathrm{C}$ in a water-saturated atmosphere. A total of 25 paired breast cancer and normal adjacent tissues were obtained from the Affiliated Drum Tower Hospital of Nanjing University Medical School (Nanjing, China); the clinical features of the patients are listed in Supplementary Table S1. All protocols concerning the use of patient samples in this study were approved by the Medical Ethics Committee of the Affiliated Gulou Hospital of Nanjing University (Nanjing, China). A signed consent form was obtained from each donor. The study protocol was approved by the Medical Ethics Committee of the Affiliated Drum Tower Hospital of Nanjing University Medical School and all the experiments were performed in accordance with the approved guidelines.
\end{abstract}

miRNA-related reagents, siRNAs and transfection. miRNA mimics, inhibitors and negative controls were purchased from GenePharma (Shanghai, China). To knock down HIC1, three siRNA sequences targeting different sites of human $\mathrm{HIC1}$ open reading frame (ORF) were designed and synthesized by GenePharma, and the sequence with the best interfering effect was selected (sequence: CCUAGUCUCCUCUAUCGCUTT). For the HIC1 overexpression assay, a mammalian expression plasmid (pReceiver-M02-HIC1) designed to specifically express the full-length ORF of human HIC1 without the miR-23 27 24responsive 3'-UTR was purchased from GeneCopoeia (Germantown, MD, USA). An empty plasmid served as a negative control. Lipofectamine 2000 (Invitrogen, Carlsbad, CA, USA) was used for transfection according to the instructions. Total RNA and protein were isolated 24 or $48 \mathrm{~h}$ after transfection.
RNA isolation and real-time quantitative PCR. Total RNA extraction, reverse transcription and TaqMan real-time polymerase chain reaction (PCR) for miRNAs were performed as described previously. ${ }^{23}$ Real-time PCR for HIC1 mRNA, C9orf3 mRNA and the sixteen intron of C9orf3 were performed using SYBR Green PCR Master Mix (Ambion, Carsbad, CA, USA). The sequences of the primers were as follows: HIC1 mRNA (sense): 5'-GTCGTGCGACAAGAGCTACAA-3'; HIC1 mRNA (antisense): 5'-CGTTGCTGTGCGAACTTGC-3'; ephrin-A1 mRNA (sense): 5'-GACTGTCAGTGGCAAAATCACTC-3'; ephrin-A1 mRNA (antisense): 5'-ACTGT GACCGATGCTATGTAGAAC-3'; SIRT1 mRNA (sense): 5'-ATCGGCTACCGAG ACAAC-3'; SIRT1 mRNA (antisense): 5'-GTCACTAGAGCTGGCGTGT-3'; C9orf3 mRNA (sense): 5'-AGGTGTTAAGACCCAGTAAAG-3'; C9orf3 mRNA (antisense): $5^{\prime}$-GAAACCGAAGAAGGAAGTAG-3'; C9orf3 the sixteen intron (sense): $5^{\prime}$-TAA GCCCTCCCTATTTCT-3'; C9orf3 the sixteen intron (antisense): $5^{\prime}-\mathrm{GACG}$ TATCACTCCCATCTA-3'; GAPDH (sense): 5'-GATATTGTTGCCATCAATGAC-3'; and GAPDH (antisense): 5'-TTGATTTTGGAGGGATCTCG-3'. The relative amount of RNA was normalized to GAPDH.

To test the expression of pre-miR-23a/b, pre-miR-24 and pre-miR-27a/b, reverse transcription PCR and real-time PCR were performed as previously described. ${ }^{24} \mathrm{The}$ sequences of the primers were as follows: pre-miR-23a/b (sense): $5^{\prime}$-CTGGGGTTC CTGGGGAT-3'; pre-miR-23a/b (antisense): 5'-TGGTAATCCCTGGCAATGTG-3'; pre-miR-24 (sense): 5'-CTCCCGTGCCTA CTGAGCT-3'; pre-miR-24 (antisense): 5'-CCCTGTTCCTGCTGAACT GAG-3'; pre-miR-27a/b (sense): 5'-GCAGGGCTTA GCTGCTTG-3'; pre-miR-27a/b (antisense): 5'-GGCGGAACTTAGCCACTGT-3'; U6 (sense): 5'-CTCGCTTCGGCAGCACA-3'; U6 (antisense): 5'-AACGCTTCACGAA TTTGCGT-3'. The relative amount of miRNA was normalized to U6.

Western blotting. Protein levels were analyzed via western blotting and performed as described previously. ${ }^{25}$ Protein levels were normalized by probing the same blots with a GAPDH antibody. The following antibodies used for western blotting were purchased from the corresponding sources: anti-HIC-1 (H-6) (Santa Cruz Biotechnology sc-271499, Santa Cruz, CA, USA), anti-SIRT1 antibody (Abcam ab32441, Cambridge, MA, USA), anti-Ephrin A1 antibody (Abcam ab124911) and anti-GAPDH (Santa Cruz Biotechnology sc-365062). Protein bands were analyzed using ImageJ software.

Luciferase reporter assay. To test the direct binding of miR-23 27 24 to the target gene HIC1, a 461-bp fragment of the HIC1 $3^{\prime}$-UTR containing the presumed miR-23a/b, miR-24 and miR-27a/b binding site was amplified by PCR with human genomic DNA as a template. The following set of primers (with restriction enzyme cut site and protection bases in the $5^{\prime}$ end) was used: CCGCT CGAGGCCTCACTGCTTCGTGCCT (forward primer) and CCCAAGCTTCTTC GCCTTCCGACTCCA (reverse primer). The two additional sequences CCGC TCGAG and CCCAAGCTT in each primer were specially added for restriction enzyme Xho I and Hind III, respectively. The PCR fragment was digested with Xho I and Hind III restriction enzyme and then inserted into the Xho I-Hind III sites of the PGL3 basic plasmid (Ambion). Successful insertion was confirmed by DNA sequencing. To test binding specificity, sequences that interacted with the miR-23a/b, miR-24 and miR-27a/b seed sequence were respectively mutated from AATGTGA to TTACACT, ACTGTGA to TGACACT, and CTGAGCC to GACTCGG and the synthetic HIC1 $3^{\prime}$-UTR mutant fragment was inserted into an equivalent reporter plasmid. For the luciferase reporter assays, 293T cells were cultured in 24-well plates, and each well was transfected with $0.1 \mu \mathrm{g}$ of firefly luciferase reporter plasmid, $0.1 \mu \mathrm{g}$ of a $\beta$-galactosidase ( $\beta$-gal) expression plasmid (Ambion) and equal amounts (100 pmol) of pre-miR-23a/b, pre-miR-24 pre-miR-27a/b or the scrambled negative control RNA using Lipofectamine 2000 (Invitrogen). The $\beta$-gal plasmid was used as a transfection control. Twenty-four hours after transfection, the cells were assayed using a luciferase assay kit (Promega, Madison, WI, USA).

In addition, to test the direct binding of HIC1 to the promoter of miR-23 27 24 clusters, three synthetic 300 bp DNA fragments (Invitrogen), which include site 1-wt (include site 1 binding sequence), site 3-wt (include site 3 binding sequence) and site 4-wt (include site 4 binding sequence) in miR-23 27 24 promoter region, were inserted into the promoter region of pGL3 basic (Ambion), and the insertion was confirmed by sequencing. To test the binding specificity, the sequences that interacted with $\mathrm{HIC} 1$ were mutated (all binding positions were mutated), and the mutant three DNA fragments were inserted into the promoter region of pGL3 basic plasmid too. 293T cells were cultured in 24-well plates, and each well was transfected with $0.1 \mu \mathrm{g}$ of firefly luciferase reporter plasmid, $0.1 \mu \mathrm{g}$ of $\beta$-gal expression plasmid (Ambion) and $0.1 \mu \mathrm{g} \mathrm{HIC1}$ overexpression plasmid or the negative control plasmid using Lipofectamine 2000 (Invitrogen). The $\beta$-gal plasmid was used as a transfection 
control. Twenty-four hours after transfection, the cells were assayed using a luciferase assay kit (Promega, Madison, WI, USA).

Cell invasion and apoptosis assays. The invasion ability and apoptosis of MCF-7 cells was tested in a Transwell Boyden Chamber $(6.5 \mathrm{~mm}$, Costar, Corning, NY, USA) and Annexin V-FITC/PI staining kit (BD Biosciences, San Diego, CA, USA) as previously described. ${ }^{25}$ Besides, the total apoptotic cells were counted as the sum of early apoptotic $(\mathrm{PI}-\mathrm{AV}+)$ and late apoptotic $(\mathrm{PI}+\mathrm{AV}+)$ cells.

Chromatin immunoprecipitation (ChIP). ChIP assays were carried out using an EZ-ChIP assay kit (Upstate Biotechnology, Inc.) in accordance with the manufacturer's instructions. Soluble chromatin was prepared from primary cortical neurons and incubated with anti-HIC1 antibody (Santa Cruz Biotechnology sc-27 1499) or with mouse IgG as a negative control. The primer pairs used for PCR analysis were as follows: for site 1: 5'-CATGGTGAAACCCCGTCT-3' (forward) and 5'-CACCTCCAGAGTTCAAGC-3' (reverse); for site 2: 5'-GGGACGGGACAGAG TTGG-3' (forward) and 5'-TTAAAACCTTCCCGTCTCCTC-3' (reverse); for site 3: 5'-AATGTGAATTGGACTGATGAC-3' (forward) and 5'-GACCAAATTACAATGACAA ACA-3' (reverse); and for site 4: 5'-ACTGGGTAGGATACAATAAG-3' (forward) and $5^{\prime}$-AAGAAAGTGGTGGCTCAT-3' (reverse). All data were normalized to the input.

Construction of miR-23a/24/27a overexpression lentiviral vector. Lentivirus for miR-23a, miR-24 or miR-27a overexpression was purchased from Invitrogen. The mature of miR-23a, miR-24 and miR-27a overexpression lentiviral vectors were mixed with equal amount and added to MCF- 7 cells at $70 \%$ confluence in six-well plates or $10-\mathrm{cm}$ dishes at an $\mathrm{MOI}$ of 10 together with polybrene at a final concentration of $5 \mu \mathrm{g} / \mathrm{ml}$ according to the manufacturer's instructions. The cells were then collected for quantitative RT-PCR, western blotting or animal experiments.

Establishment of tumor xenografts in mice. Six-week-old female SCID (severe combined immune deficiency) mice (nu/nu) were purchased from the Model Animal Research Center of Nanjing University (Nanjing, China) and maintained under specific pathogen-free conditions at Nanjing University. Control MCF-7 cells, miR-23a/24/27a-overexpressing MCF-7 cells, HIC1- overexpressing MCF-7 cells or miR-23a/24/27a and HIC1 co-overexpressing MCF-7 cells were injected subcutaneously into SCID mice $\left(2 \times 10^{6}\right.$ cells per mouse, six mice per group). Tumor volume was measured every 3 days for 21 days after inoculation. The length, width and height of the tumors were measured with digital calipers and the ellipsoid volume was calculated using the following formula: Volume $=\pi / 6 \times($ length $) \times$ (width) $\times$ (height). The tumor tissue was fixed in $10 \%$ formalin for $24 \mathrm{~h}$ and then further processed for H\&E staining and for immunohistochemical staining for Ki-67 and HIC1. The whole experimental procedure was repeated three times. All animal care and handling procedures were performed in accordance with the National Institutes of Health's Guide for the Care and Use of Laboratory Animals and were approved by the Institutional Review Board of Nanjing University (Nanjing, China).

Mathematical models. We first refined the existing mathematical models of double-negative feedback loops $s^{26,27}$ and miRNAs ${ }^{28}$ to create a system of two nonlinear ordinary differential equations (ODEs) using fundamental biochemical laws and parameters extracted from the literature. ${ }^{28-31}$ These equations described the time dependences of $\mathrm{HIC1}$ protein $(\mathrm{p})$ and $\mathrm{miR}-24(\mathrm{~m})$ concentrations based on the rates of synthesis and degradation of these molecules as controlled by their mutual repression and inhibition. The original equations were then converted into a single equation that could be solved for the steady state of the system by eliminating one variable with time derivatives of ODEs set to 0 . We generated bifurcation diagrams ${ }^{28}$ using Matlab (The MathWorks, Inc.) to graphically analyze the steady states (converted to relative levels) of the system by changing the synthesis rate of HIC1 protein and miR-24 over a range so that the impact of epigenetic downregulation of $\mathrm{HIC} 1$ and transfection of mimic-miR-24 on the system could be mimicked and illustrated. A detailed description about this mathematical modeling is shown in Supplementary Methods.

Statistical analysis. All western blot images are representative of at least three independent experiments. Quantitative RT-PCR, luciferase reporter assay, chromatin immunoprecipitation, cell invasion assay and cell apoptosis assay were performed in triplicate, and each experiment was repeated several times. The data shown are the mean \pm S.E. of at least three independent experiments. Differences were considered statistically significant at $P<0.05$ using Student's $t$-test.

\section{Conflict of Interest}

The authors declare no conflict of interest.

Acknowledgements. We thank Professor Jun Cui (Sun Yat-sen University, Guangzhou) for helpful discussion in building mathematical modeling. This work was supported by grants from the National Basic Research Program of China (973 Program) (No. 2014CB542300), the National Natural Science Foundation of China (No. 31271378, J1103512 and J1210026), the Research Special Fund for Public Welfare Industry of Health (No. 201302018) and the Natural Science Foundation of Jiangsu Province (No. BE2016737).

1. Torre LA, Bray F, Siegel RL, Ferlay J, Lortet-Tieulent J, Jemal A. Global Cancer Statistics, 2012. CA Cancer J Clin 2015; 65: 87-108.

2. DeSantis C, Siegel R, Bandi $P$, Jemal A. Breast cancer statistics2011 CA Cancer J Clin 2011; 61: 409-418

3. Shi M, Guo N. MicroRNA expression and its implications for the diagnosis and therapeutic strategies of breast cancer. Cancer Treat Rev 2009; 35: 328-334.

4. Andorfer CA, Necela BM, Thompson EA, Perez EA. MicroRNA signatures: clinical biomarkers for the diagnosis and treatment of breast cancer. Trends Mol Med 2011; 17: 313-319.

5. O'Day E, Lal A. MicroRNAs and their target gene networks in breast cancer. Breast Cancer Res 2010; 12: 2.

6. Ma L, Weinberg RA. Micromanagers of malignancy: role of microRNAs in regulating metastasis. Trends Genet 2008; 24: 448-456.

7. Nicoloso MS, Spizzo R, Shimizu M, Rossi S, Calin GA. MicroRNAs - the micro steering wheel of tumour metastases. Nat Rev Cancer 2009; 9: 293-302.

8. Griffiths-Jones S, Saini HK, van Dongen S, Enright AJ. miRBase: tools for microRNA genomics. Nucleic Acids Res 2008; 36: D154-D158.

9. Lee Y, Jeon K, Lee JT, Kim S, Kim VN. MicroRNA maturation: stepwise processing and subcellular localization. EMBO J 2002; 21: 4663-4670.

10. Chhabra R, Dubey R, Saini N. Cooperative and individualistic functions of the microRNAs in the miR-23a similar to 27a similar to 24-2 cluster and its implication in human diseases. Mol Cancer 2010; 9: 232.

11. Ozsolak F, Poling LL, Wang Z, Liu H, Liu XS, Roeder RG et al. Chromatin structure analyses identify miRNA promoters. Genes Dev 2008; 22: 3172-3183.

12. Martinez NJ, Walhout AJM. The interplay between transcription factors and microRNAs in genome-scale regulatory networks. Bioessays 2009; 31: 435-445.

13. Lewis BP, Shih IH, Jones-Rhoades MW, Bartel DP, Burge CB. Prediction of mammalian microRNA targets. Cell 2003; 115: 787-798.

14. John B, Enright AJ, Aravin A, Tuschl T, Sander C, Marks DS. Human MicroRNA targets. PLOS Biol 2004; 2: 1862-1879.

15. Krek A, Grun D, Poy MN, Wolf R, Rosenberg L, Epstein EJ et al. Combinatorial microRNA target predictions. Nat Genet 2005; 37: 495-500.

16. Cheng G, Sun X, Wang J, Xiao G, Wang X, Fan X et al. HIC1 silencing in triple-negative breast cancer drives progression through misregulation of LCN2. Cancer Res 2014; 74: 862-872

17. Chen WY, Wang DH, Yen RWC, Luo JY, Gu W, Baylin SB. Tumor suppressor HIC1 directly regulates SIRT1 to modulate p53-dependent DNA-damage responses. Cell 2005; 123 : 437-448.

18. Pinte S, Stankovic-Valentin N, Deltour S, Rood BR, Guerardel C, Leprince D. The tumor suppressor gene $\mathrm{HIC1}$ (Hypermethylated in cancer 1) is a sequence-specific transcriptional repressor - Definition of its consensus binding sequence and analysis of its DNA binding and repressive properties. J Biol Chem 2004; 279: 38313-38324.

19. Zhang W, Zeng X, Briggs KJ, Beaty R, Simons B, Yen RWC et al. A potential tumor suppressor role for Hic1 in breast cancer through transcriptional repression of ephrin-A1. Oncogene 2010; 29: 2467-2476.

20. Ferrell JE. Self-perpetuating states in signal transduction: positive feedback, double-negative feedback and bistability. Curr Opin Cell Biol 2002; 14: 140-148.

21. Lee J, Lee J, Farquhar KS, Yun J, Frankenberger CA, Bevilacqua E et al. Network of mutually repressive metastasis regulators can promote cell heterogeneity and metastatic transitions. Proc Natl Acad Sci USA 2014; 111: E364-E373.

22. Brock A, Chang H, Huang S. OPINION Non-genetic heterogeneity - a mutationindependent driving force for the somatic evolution of tumours. Nat Rev Genet 2009; 10: 336-342.

23. Zou Z, Wu L, Ding H, Wang Y, Zhang Y, Chen X et al. MicroRNA-30a sensitizes tumor cells to cis-platinum via suppressing beclin 1-mediated autophagy. J Biol Chem 2012; 287: 4148-4156

24. Schmittgen TD, Jiang JM, Liu Q, Yang LQ. A high-throughput method to monitor the expression of microRNA precursors. Nucleic Acids Res 2004; 32: e43.

25. Liang H, Liu M, Yan X, Zhou Y, Wang W, Wang X et al. miR-193a-3p functions as a tumo suppressor in lung cancer by down-regulating ERBB4. J Biol Chem 2015; 290: 926-940.

26. Gardner TS, Cantor CR, Collins JJ. Construction of a genetic toggle switch in Escherichia coli. Nature 2000; 403: 339-342. 
27. Kramer BP, Viretta AU, Daoud-El-Baba M, Aubel D, Weber W, Fussenegger M. An engineered epigenetic transgene switch in mammalian cells. Nat Biotechnol 2004; 22: 867-870.

28. Aguda BD, Kim Y, Piper-Hunter MG, Friedman A, Marsh CB. MicroRNA regulation of a cancer network: consequences of the feedback loops involving miR-17-92, E2F, and Myc. Proc Natl Acad Sci USA 2008; 105: 19678-19683.

29. Zhou $\mathrm{CH}$, Zhang XP, Liu F, Wang W. Involvement of miR-605 and miR-34a in the DNA damage response promotes apoptosis induction. Biophys $J$ 2014; 106: 1792-1800.

30. Bartlett DW, Davis ME. Insights into the kinetics of siRNA-mediated gene silencing from livecell and live-animal bioluminescent imaging. Nucleic Acids Res 2006; 34: 322-333.

31. Milo R, Jorgensen P, Moran U, Weber G, Springer M. BioNumbers-the database of key numbers in molecular and cell biology. Nucleic Acids Res 2010; 38(Database issue): D750-D753. (c) (i) $\$$ This work is licensed under a Creative Commons Attribution-NonCommercial-NoDerivs 4.0 International License. The images or other third party material in this article are included in the article's Creative Commons license, unless indicated otherwise in the credit line; if the material is not included under the Creative Commons license, users will need to obtain permission from the license holder to reproduce the material. To view a copy of this license, visit http://creativecommons.org/licenses/by-nc-nd/4.0/

(C) The Author(s) 2017

Supplementary Information accompanies this paper on Cell Death and Differentiation website (http://www.nature.com/cdd) 